\title{
Phenotypic variability and heterozygosity at an esterase locus in the mosquito Aedes aegypti
}

\author{
F. K. Kasule* and \\ L. M. Cook
}

Department of Environmental Biology, University of Manchester, Manchester M13 9PL, U.K.

Populations of the yellow fever mosquito, Aedes aegypti L. are polymorphic for esterase alleles. This could be because the alleles are neutral or because selection protects the polymorphism, as had been concluded from a study of allele frequencies. A test has been carried out to look for possible associations of esterase heterozygosity with speed of development, body size and spine number symmetry in two spine rows. Heterozygotes were found to be larger and slower growing than homozygotes, but there was no difference in degree of asymmetry. There were fewer heterozygotes and a greater deficiency compared with expectation in insects reared at higher temperatures. It is concluded that different genotypes do differ in fitness.

\section{INTRODUCTION}

When kept as laboratory stocks, colonies of the yellow fever mosquito, Aedes aegypti are polymorphic for esterase alleles, as they are in the wild. One possible reason for this is that the alleles are neutral, the effective population size in the wild is sufficiently large that the insects taken from it are very likely to be heterozygous for different pairs of alleles and that insufficient time has elapsed for the polymorphism to be lost in the laboratory. The other is that although stochastic fluctuations may be involved, the polymorphisms are maintained by selection. Studies made by Saul, Guptavanij and Craig (1976) and by Mani, Cook and Marvdashti (1986) showed that laboratory stocks have an average of two to three alleles at the esterase six locus, even after many generations of culture in artificial conditions. The pattern of allele frequency distribution makes it unlikely that they are entirely neutral (Mani et al., 1986). Different stocks are polymorphic for different combinations of alleles. If selection is to be invoked to account for this variability, however, the question naturally arises what kind of selection could protect different sets of alleles in different stocks living under the same artificial conditions in the laboratory. One

\footnotetext{
* Present address: Department of Zoology, University of Dar Es Salaam, PO Box 35064, Dar Es Salaam, Tanzania.
}

possibility is that heterozygosity, either at the esterase locus itself or in one or more loci linked to it and in linkage disequilibrium, increases the general fitness of the heterozygous individuals. Many studies have now been recorded (Mitton and Grant, 1984; Zouros and Foltz, 1987; Zouros, 1987; Livshits and Kobyliansky, 1985) in which an association has been shown between heterozygosity at enzyme loci and characters such as speed of development, size and bilateral symmetry. Sometimes the associations are not shown (e.g., McAndrew et al., 1982; Strauss and Libby, 1987) or are negative (Diehl and Koehn, 1985; Ledig et al., 1983), but it is probable that non-positive results are under-represented in the literature. We therefore considered the possibility that $A$. aegypti heterozygous for esterase alleles would score more highly than homozygotes for fitness traits. If so, we would expect the association to be with heterozygosity as such rather than with a particular allele combination.

The larvae have two pairs of spine rows, the comb and pecten, at the posterior end of the abdomen. Spine number is known to be heritable, shows a degree of bilateral asymmetry, and in the case of the pecten, is subject to stabilizing selection during development (Wood, 1976a, b; Wood and Dalingwater, 1975). It is therefore possible that individuals symmetrical for spine numbers have higher fitness than asymmetrical ones. Similarly, 
body size and speed of development may be related to general fitness. We have measured these characters in individuals homozygous or heterozygous at the variable esterase locus in order to examine whether associations exist.

\section{MATERIALS AND METHODS}

The stocks used were: a newly colonized one from Venezuela, two which had been maintained for many generations in the laboratory and came from Bangalore, India, and Trinidad respectively, and a line called HP, derived in Manchester by mixing a number of different laboratory strains (Walker and Wood, 1986). Adults were fed and mass mated, and eggs collected at $28^{\circ} \mathrm{C}$. These were hatched and raised at $28^{\circ} \mathrm{C}$ in the first trial, at the optimal temperature for the species. Two other sets were raised at $32^{\circ} \mathrm{C}$ and $35^{\circ} \mathrm{C}$ respectively in order to apply greater stress $t$ the developing larvae. Two replicate batches of larvae were raised from each strain, and scored for number of spines in left and right comb and pecten rows. The time from emergence to pupation was recorded in units of $6 \mathrm{hr}$, and the length of the pupa in graticule units. The individual pupae were then killed and scored for esterase bands using vertical slab polyacrylamide gel electrophoresis. The original intention was to raise about 100 larvae from each strain in each series, but mortality during development reduced the number raised at the higher temperatures. Some additional batches were therefore scored at $35^{\circ} \mathrm{C}$.

Palmer and Strobeck (1986) investigated the efficiency of methods for detecting associations of asymmetry with heterozygosity. They concluded that the most sensitive approach is to calculate and compare the mean squared deviations for each class. These then have to be tested as variance ratios. The other two measures, speed of development and body size, may be compared between groups using the $t$-test.

The hypothesis we wish to test is that heterozygous individuals have one or all of the following characters compared with homozygotes: greater bilateral symmetry, higher speed of development, and larger size. There is no a priori reason to choose one of these measures over the others as a measure of fitness. The correlation coefficients between the variables are quite low (see below), and we have therefore examined all three. The reciprocal of the duration of the larval stage $(\times 100)$ is used as a measure of speed of development. Growth rate and size are compared between genotype classes by calculating $t$, which for the numbers of degrees of freedom involved is virtually a normal deviate. To compare the mean squared deviations of bilateral differences in spine number we have used Bartlett's test for homogeneity of variance to provide $\chi^{2}$ values with 1 degree of freedom, and the signed square roots of these values, which are normal deviates. Bartlett's test tends to over-estimate significance due to non-normal distribution but trial values for the two-sample case gave good agreement whether tested as $\chi^{2}$ or variance ratio. Tests between groups within strains and between strains indicated no heterogeneity, so that the results presented here are for the pooled data.

\section{RESULTS}

A summary of the results is given in table 1, which shows mean and variance for speed of development and body size in each class, and variance for asymmetry of comb and pecten number. Table 2 gives the normal deviates calculated for each pairwise comparison. Negative values for speed of development and size indicate that heterozygotes grew more slowly or were smaller than homozygotes; negative values for comb and pecten indicate greater asymmetry in the heterozygotes. The bottom row of table 2 gives the overall normal deviate, obtained by summing the columns and dividing by the number of rows. There is no tendency for comb or pecten to differ in symmetry between esterase classes, but speed of development is significantly lower in heterozygotes than in homozygotes and the size attained is significantly greater $(P<0.05)$.

If we reject the significant negative association of speed of development with heterozygosity because it is negative, then one wonders whether the significant association with size should be accepted merely because it is positive. On the other hand, we may treat the four comparisons as twotailed tests exploring possible relationships, and combine the probabilities using Fisher's method. The overall result is significantly different from random expectation on this hypothesis $\left(\chi_{8}^{2}=17 \cdot 8\right.$, $P<0.025)$, and esterase heterozygotes are larger and more slow-growing than homozygotes. This would suggest that the esterase alleles do have some differential effects on their carriers.

Although the level of association is low, size and speed of development are positively correlated in the whole data set (table 3 ), so that the test above is overestimating the significance of the overall effect. The last column of table 3 shows the 
Table 1 Mean and variance for speed of development and body size and variance of difference between left and right sides for comb and pecten spine numbers in samples of Aedes aegypti larvae. At each rearing temperature and in each sex, samples heterozygous or homozygous at an esterase locus are compared. Speed of development is the reciprocal of development time in $\mathrm{hr} \times 100$. Size is given in graticule units

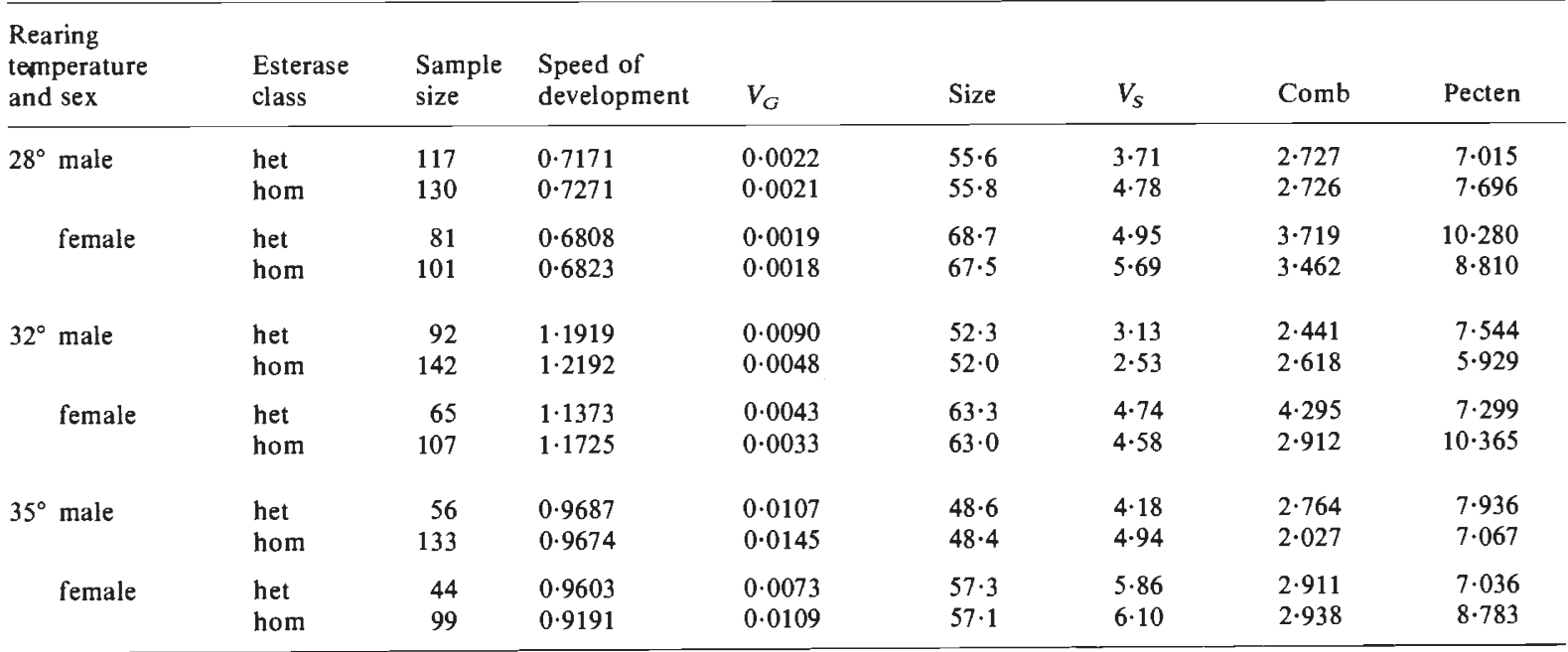

normal deviates testing the deviation of the correlation coefficients from zero. If heterozygotes are both larger and slower growing than homozygotes, then they should show a lower level of correlation. The combined normal deviate for heterozygotes is $1.57(P>0.05)$, compared with $4.58(P<0.001)$ for homozygotes. This test therefore also indicates a difference in growth pattern between individuals with different esterase allele combinations.

There is clear systematic effect when results at different temperatures are compared. The association between size and speed of development increases with increasing temperature (table 3 ). Also, the sample sizes listed in table 1 show that

Table 2 Normal deviates for differences between heterozygotes and homozygotes for the characters shown in table 1. Negative values indicate that the value is smaller (growth rate and body size) or more asymmetrical (comb and pecten) in heterozygotes. The pooled values are obtained by summing the columns and dividing by the square root of the number of rows. $27,32,35$ : rearing temperature; $m, f$ : sex

\begin{tabular}{lrrrr}
\hline & $\begin{array}{l}\text { Growth } \\
\text { rate }\end{array}$ & $\begin{array}{l}\text { Body } \\
\text { size }\end{array}$ & $\begin{array}{l}\text { Asymmetry } \\
\text { comb }\end{array}$ & $\begin{array}{l}\text { Asymmetry } \\
\text { pecten }\end{array}$ \\
\hline $28 \mathrm{~m}$ & -1.69 & -0.42 & -0.003 & 0.51 \\
$28 \mathrm{f}$ & -0.23 & 3.44 & -0.34 & -0.73 \\
$32 \mathrm{~m}$ & -2.38 & 1.47 & 0.37 & -1.28 \\
$32 \mathrm{f}$ & -3.57 & 0.75 & -1.76 & 1.54 \\
$35 \mathrm{~m}$ & 0.08 & 0.40 & -1.39 & -0.52 \\
$35 \mathrm{f}$ & 2.48 & 0.45 & 0.04 & 0.84 \\
Pooled & -2.17 & 2.49 & -1.26 & 0.15 \\
\hline
\end{tabular}

the ratios of heterozygotes to homozygotes decrease with increase in temperature. There is no difference in the ratio between sexes at each temperature. When the sexes are combined, the decline in frequency of heterozygotes from $28^{\circ} \mathrm{C}$ to $32^{\circ} \mathrm{C}$ $\left(\chi_{1}^{2}=4 \cdot 78\right)$ and from $32^{\circ} \mathrm{C}$ to $35^{\circ} \mathrm{C}\left(\chi_{1}^{2}=5 \cdot 88\right)$ are both significant $(P<0.05)$. Not only are there fewer heterozygotes in insects raised at higher temperatures, but their deficiency compared with expectation also changes. Table 4 gives the

Table 3 Correlation between body size and speed of development. The last column, $d$, shows normal deviates measuring deviation of the correlation coefficient, $r$, from zero

\begin{tabular}{|c|c|c|c|c|c|}
\hline \multicolumn{2}{|c|}{$\begin{array}{l}\text { Rearing } \\
\text { temperature } \\
\text { and sex }\end{array}$} & \multirow{3}{*}{$\begin{array}{l}\begin{array}{l}\text { Esterase } \\
\text { class }\end{array} \\
\text { het } \\
\text { hom }\end{array}$} & \multirow{3}{*}{$\begin{array}{l}\begin{array}{l}\text { Sample } \\
\text { size }\end{array} \\
117 \\
130\end{array}$} & \multirow{3}{*}{$\begin{array}{r}r \\
-0.068 \\
0.203\end{array}$} & \multirow{3}{*}{$\begin{array}{r}d \\
-0 \cdot 73 \\
2 \cdot 32\end{array}$} \\
\hline \multirow[t]{4}{*}{$28^{\circ}$} & male & & & & \\
\hline & & & & & \\
\hline & female & het & 81 & $-0 \cdot 125$ & $-1 \cdot 11$ \\
\hline & & hom & 101 & 0.122 & $1 \cdot 21$ \\
\hline \multirow[t]{4}{*}{$32^{\circ}$} & male & het & 92 & 0.145 & $1 \cdot 38$ \\
\hline & & hom & 142 & $0 \cdot 113$ & $1 \cdot 34$ \\
\hline & female & het & 65 & $0 \cdot 157$ & $1 \cdot 25$ \\
\hline & & hom & 107 & $0 \cdot 120$ & $1 \cdot 23$ \\
\hline \multirow[t]{4}{*}{$35^{\circ}$} & male & het & 56 & 0.285 & $2 \cdot 13$ \\
\hline & & hom & 133 & 0.219 & $2 \cdot 54$ \\
\hline & female & het & 44 & 0.142 & 0.92 \\
\hline & & hom & 99 & 0.257 & $2 \cdot 57$ \\
\hline
\end{tabular}


Table 4 Observed heterozygosity as a fraction of expected heterozygosity, and its standard error in four stocks raised at three temperatures

\begin{tabular}{llll}
\hline \multirow{4}{*}{ Stock } & \multicolumn{2}{l}{ Rearing Temperature } \\
\cline { 2 - 4 } & $28^{\circ} \mathrm{C}$ & $32^{\circ} \mathrm{C}$ & $35^{\circ} \mathrm{C}$ \\
\hline Venezuela & $1.066 \pm 0.065$ & $0.957 \pm 0.104$ & $0.669 \pm 0.106$ \\
Bangalore & $0.577 \pm 0.085$ & $0.610 \pm 0.078$ & $0.459 \pm 0.096$ \\
Trinidad & $0.507 \pm 0.090$ & $0.450 \pm 0.093$ & $0.392 \pm 0.108$ \\
HP & $0.814 \pm 0.072$ & $0.891 \pm 0.081$ & $0.636 \pm 0.081$ \\
\hline
\end{tabular}

observed heterozygosity as a fraction of that expected, calculated as the complement of the sum of squares of allele frequencies. There are four alleles present in the first three strains, three in the last. In the Venezuela and Bangalore strains the same allele rises in frequency, in Trinidad it remains more or less constant and in HP it is absent. The locus is therefore subject to selection under some laboratory conditions, and different alleles are favoured in different strains.

\section{DISCUSSION}

The experiment set out to test for an association between heterozygosity at an esterase locus and fitness-related characters, because it seemed possible that this would give a clue to the way the esterase polymorphism is maintained. Chakraborty (1987) has questioned whether associations of this kind, when they occur, indicate the existence of overdominance, and indeed they may not do so.

It is remarkable enough, however, that enzyme loci, picked essentially at random, ever predict fitness-related characters. That they do so implies either that most enzyme loci have a high probability of influencing general fitness directly or that they identify segments of chromosomes in linkage disequilibrium which do so. Koehn et al. (1988) examined a situation where loci with large effects were a class with specific effects on metabolism. It is important to know how likely it is that such associations will be found, so that negative data are valuable, as well as positive.

The results obtained do not provide unequivocal evidence for such an association. We originally thought that symmetry was a promising variable to study (Mani et al., 1986). There is no association of heterozygosity with symmetry, and the association with speed of development is not in the direction originally predicted. The results do not allow us to speculate on how the polymorphism may be protected. Nevertheless, they do demonstrate the existence of selection, so that the polymorphism cannot be interpreted on the assumption of complete neutrality. They therefore support the conclusion of Mani et al. (1966) derived from a study of the allele frequency distribution.

Acknowledgements We thank R. J. Wood for providing stocks and rearing facilities and G. S. Proudlove for technical help. The work was carried out while Kasule held a Commonwealth Academic Staff Fellowship. We are grateful to G. S. Mani, R. J. Wood and a referee for helpful comments.

\section{REFERENCES}

CHAKRABORTY, R. 1987. Biochemical heterozygosity and phenotypic variability of polygenic traits. Heredity, 59, 19-28.

DIEHL, W. J. AND KOEHN, R. K. 1985. Multiple-locus heterozygosity, mortality and speed of development in a cohort of Mytilus edulis. Mar. Biol., 88, 265-271.

KOEHN, R. K., DIEHL, W. J. AND SCOTT, T. M. 1988. The differential contribution by individual enzymes of glycolysis and protein catabolism to the relationship between heterozygosity and speed of development in the coot clam, Mulinia lateralis. Genetics, 118, 121-130.

LEDIG, F. T., JURIES, R. P. AND BONEFELD, B. A. 1983. The relation of growth to heterozygosity in pitch pine. Evolution, 37, 1227-1238.

LIVSHITS, G. AND KOBYLIANSKY, E. 1985. Lerner's concept of developmental homeostasis and the problems of heterozygosity levels in natural populations. Heredity, 55, 341-353.

McANDREW, B. J., WARD, R. D. AND BEARDMAN, J. A. 1982. Lack of relationships between morphological variance and enzyme heterozygosity in the plaice, Pleuronectes plaseisa. Heredity, 48, 117-125.

MANI, G. S., COOK, L. M. AND MARVDASHTI, R. 1986. What can be learnt about selection from gene frequency distributions? Genetics, 114, 971-982.

MITTON, J. B. AND GRANT, M. C. 1984. Association among heterozygosity, growth rate and developmental homeostasis. Ann. Rev. Ecol. Syst., 15, 479-499.

PALMER, A. R. AND STROBECK, C. 1986. Fluctuating asymmetry: measurement, analysis, patterns. Ann. Rev. Ecol. Syst., 17, 391-421.

SAUL, S. H., GUPTAVANIJ, P. AND CRAIG, G. B. 1976. Genetic variability at an esterase locus in Aedes aegypti. Ann. Entomol. Soc. America, 69, 73-79.

STRAUSS, S. H. AND LIBBY, w. J. 1987. Allozyme heterosis in radiata pine is poorly explained by overdominance. Amer. Natur. 130, 879-890

WALKER, A. L. AND WOOD, R. J. 1986. Laboratory selected resistance to diflubenzuron in larvae of Aedes aegypti. Pestic. Sci., 17, 495-502.

wOOD, R. J. $1976 a$. Spine numbers on the pecten and comb of fourth instar larvae of Aedes aegypti L. Differences between two populations. Genetica, 46, 33-44. 
WOOD, R. J. $1976 b$. The effect of DDT and rearing temperature on pecten spine number in larvae of the mosquito Aedes aegypti L. Genetica, 46, 45-48.

WOOD, R. J. AND DALINGWATER, J. E. 1975. Changes in comb spine number during larval development in Aedes aegypti L. Mosquito News, 35, 555-560.
zOUROS, E. 1987. On the relation between heterozygosity and heterosis: an evaluation of the evidence from marine molluscs. Isozymes. Curr. Top. Biol. Med. Res., 15, 255-270.

ZOUROS, E. AND FOLTZ, D. W. 1987. The use of allele isozyme variation for the study of heterosis. Isozymes. Curr. Top. Biol. Med. Res., 13, 1-59. 